\title{
Numerical investigation of the influence of pattern topology on the mechanical behavior of PEGDA hydrogels
}

\author{
Tao Jin ${ }^{\mathrm{a}}$, Ilinca Stanciulescu ${ }^{\mathrm{a}, *}$ \\ ${ }^{a}$ Department of Civil and Environmental Engineering, Rice University, Houston, TX \\ 77005, USA
}

\begin{abstract}
Poly(ethylene glycol) diacrylate (PEGDA) hydrogels can potentially be used as scaffold material for tissue engineered heart valves (TEHVs) due to their good biocompatibility and biomechanical tunability. The photolithographic patterning technique is an effective approach to pattern PEGDA hydrogels to mimic the mechanical behavior of native biological tissues that are intrinsically anisotropic. The material properties of patterned PEGDA hydrogels largely depend on the pattern topology. Currently, designing patterned PEGDA hydrogels that meet targeted material properties is still a trial-and-error process. Depending on experiments alone to explore the influence of pattern topology is expensive and time-consuming. In this paper, we adopt a newly proposed computational framework for fibrous biomaterials to numerically investigate the influences of pattern topology, including pattern ratio, orientation and waviness, on the mechanical behavior of patterned PEGDA hydrogels. The material parameters for the base hydrogel and the pattern stripes are directly calibrated from published experimental data. Several experimental observations reported in literature are captured in the simulations, including the nonlinear relationship between pattern ratio and material linear modulus, and the decrease of material anisotropy when pattern ratio increases. We further numerically demonstrate
\end{abstract}

\footnotetext{
${ }^{*}$ Corresponding author

Email address: ilinca.s@rice.edu (Ilinca Stanciulescu)
}

Preprint submitted to Journal of ${ }^{A} T_{E} X$ Templates

October 10, 2016

(C) 2016. This manuscript version is made available under the Elsevier user license http://www.elsevier.com/open-access/userlicense/1.0/ 
that a three-region (toe-heel-linear) stress-strain relationship typically exhibited by biological tissues can be obtained by tuning the pattern geometry and the material properties of the base hydrogel and the pattern stripes. The numerical strategy and simulation results presented in this paper can provide guidance to optimize the pattern design of PEGDA hydrogels toward the targeted material properties, therefore advance the development of TEHVs.

Keywords: PEGDA hydrogel, Pattern topology, Finite Element Method

\section{Introduction}

Valvular heart disease involves the malfunction of one or more of the four heart valves. The symptoms range from moderate palpitation to lethal heart failure. According to a recent study, an estimate of 4.2 to 5.6 million adults

5 in the U.S. have valvular diseases [1]. Currently, the preferred method to treat heart valve patients is to repair the diseased valves. However, when the valve disease is severe, just repairing is not sufficient and therefore replacement is required [2]. Each year nearly 300,000 valve replacement surgeries are performed worldwide, and this number is predicted to triple in the next 30 years due to 10 the increase of the aging population $[2,3]$.

Nowadays there are two basic types of artificial valves that are clinically available for replacement, mechanical valves and bioprosthetic valves. Mechanical valves are made of strong durable materials, and in most cases can last throughout the remainder of the patient's lifetime. However, clot-risks are high for patients with this type of valves. Consequently, lifelong treatment with blood thinner is required to keep clots from forming. On the other hand, bioprosthetic valves are created from animal valves, and do not require long-term use of medication. But their relatively short lifespan (from 10 to 20 years) increases the likelihood of extra surgeries. Biocompatibility is another issue related with bio20 prosthetic valves, since human bodies tend to reject foreign materials. Moreover, a major disadvantage of both types is that valve tissue growth and remodeling are not allowed after implantation. This is a significant drawback especially for 
young patients, whose heart valves must grow over time [4]. With the recent advance in tissue engineering, tissue engineered heart valves (TEHVs) provide a promising potential to generate suitable heart valve replacements $[5,6,7,8,9]$.

TEHVs usually require biodegradable scaffolds to encapsulate cells for tissue growth and remodeling, and simultaneously must provide sufficient mechanical functions after implantation, which is crucial for the survival of the patient [4]. The scaffolds for the most successful TEHVs to date are non-woven fiber mesh or salt-leached foam scaffolds generated from polyglycolic acid and polyhydroxyalkanoates $[10,11,12]$. However, these materials have certain limitations, including: (1) high initial stiffness compared with native tissues; (2) long time required for the polymer to undergo degradation [13]; (3) lack of intrinsic biological functionalization [6]. Because of the biochemical and biomechanical tunabil35 ity, poly(ethylene glycol) diacrylate (PEGDA) hydrogels have received more and more attention to be used as scaffold materials for TEHVs $[14,15,16,6,7,8]$. PEGDA hydrogel is a soft bio-inspired material that possesses many important features, among which are biocompatibility, resistance to protein absorption, and tunable biochemical and biomechanical properties [17, 16]. Among its various biomedical applications $[18,19,8]$, PEGDA hydrogel is being investigated as a scaffold material to fabricate TEHVs.

From the mechanical perspective, hydrogels are isotropic materials, possessing elastic moduli from around $1 \mathrm{kPa}$ [20] to above $100 \mathrm{kPa}[21,22,9]$. In order to mimic the mechanical behavior of native heart valve tissues that are intrinsically anisotropic [23], advanced patterning and fabrication technologies are required. For example, using photolithographic patterning technology, PEGDA hydrogels can be three dimensionally patterned to exhibit desired anisotropic mechanical behavior $[15,14,16,9]$. In this process, the microscale characteristics, such as crosslinking density and polymer chain length, can also be tailored. More 50 recently, confocal images generated from native tissues are utilized to guide twophoton laser scanning lithography to pattern hydrogel scaffolds, and the original tissue images and generated hydrogel polymer networks are matched with good fidelity [24]. 
At the microscopic level, hydrogels are networks of crosslinked polymers and solvent molecules $[17,15,14,18]$ that can be modeled as fiber networks. The microscopic parameters, such as crosslinking density, fiber density and fiber chain length [25, 26, 27], largely influence the material macro-mechanical behavior. At the macroscopic level, it would be desirable to have a patterned PEGDA hydrogel that is a nonlinear, anisotropic material, and can sustain large deformations. At the same time, the mechanical properties of patterned PEGDA hydrogels should have the capability to be tuned by pattern topology.

In literature, native biological tissues are widely reported to exhibit a stressstrain curve that can be divided into three regions, a toe region followed by a heel region and a linear region $[28,20,29,4]$. The toe region shows a relatively low elastic modulus due to the fact that most collagen fibers are in the crimp state and do not contribute to the material stiffness at small tensile strains. As the strain increases, the collagen fibers start to be straightened, leading to the upward bend of the stress-strain curve in the heel region. The linear region exhibits a significantly larger linear modulus, since most collagen fibers are fully straightened and contribute to the total material stiffness. In order to mimic the mechanical behavior of native biological tissues, it is important to properly design the pattern topology so that hydrogels can exhibit a similar three-region stress-strain relationship.

Although many efforts have been devoted to study the material property 75 of hydrogels in recent years, the structure-function relationship of patterned PEGDA hydrogel has not yet been fully understood. Consequently, designing patterned PEGDA hydrogels that meet specific functional requirements is still a trial-and-error process. Depending on experiments alone to investigate the influence of pattern topology is time-consuming and expensive. On the other hand, numerical methods, especially the finite element method (FEM), combined with experiments, can provide better descriptions of PEGDA hydrogels with complex geometric patterns, and eventually facilitate the material design process.

In this paper, we adopt a newly proposed computational framework [30] to 
numerically investigate the influence of pattern topology, including pattern ratio, orientation, and waviness, on the mechanical behavior of patterned PEGDA hydrogels. The material parameters used in the FE simulations are calibrated from published experimental data [9]. The nonlinear relationship between the pattern ratio and the hydrogel stiffness, which is a physical phenomenon reported by different researchers [16, 9], is successfully recovered. More importantly, the typical toe-heel-linear (three-region) shape of the stress-strain relationship is also captured in the numerical simulation by properly tuning the hydrogel properties. The numerical strategy and simulation results presented in this research can provide helpful guidances toward designing the pattern geom-

95 etry of PEGDA hydrogels to mimic the targeted behavior of native heart valve tissues.

\section{Methods}

In this section, the photolithographic patterning technique $[16,9]$ used to pattern PEGDA hydrogel is briefly reviewed. The numerical strategy adopted by this paper is also introduced. Based on this strategy, the material parameters used in FE simulations are calibrated from the published experimental data. Using the calibrated material parameters, the influence of pattern geometry on the mechanical behavior of patterned PEGDA hydrogels is thoroughly investigated.

\subsection{Photolithographic patterning}

Photolithographic patterning techniques are widely used to pattern PEGDA hydrogels. Here, the patterning process adopted in $[16,9]$ is briefly described. PEGDA hydrogel with a high molecular weight (MW), in this case 20 kilodalton $(\mathrm{kDa})$, was prepared as the base hydrogel by crosslinking under the collimated ${ }_{110}$ UV light. Next, the base hydrogel was soaked in the $3.4 \mathrm{kDa}$ PEGDA solution to allow the low MW PEGDA diffuse into the high MW polymer network. Then, the hydrogel sample covered with a pre-designed photomask was exposed 
under the collimated UV light. In the hydrogel under the transparent area of the photomask, $3.4 \mathrm{kDa}$ PEGDA molecules were crosslinked into the $20 \mathrm{kDa}$ base hydrogel due to the exposure under the collimated UV light. Finally, the patterned hydrogel was soaked in the phosphate buffered saline (PBS) to remove the unreacted PEGDA molecules. Fig. 1 shows a sample of patterned PEGDA hydrogel with $20 \%$ pattern ratio. We refer to the base hydrogel soaked in the $3.4 \mathrm{kDa}$ PEGDA solution and completely exposed under the UV light without using a photomask as the $100 \%$ pattern. For the detailed PEGDA fabrication and patterning process, interested readers are referred to Fig. 1 in [16] or Fig. 2 in $[9]$.

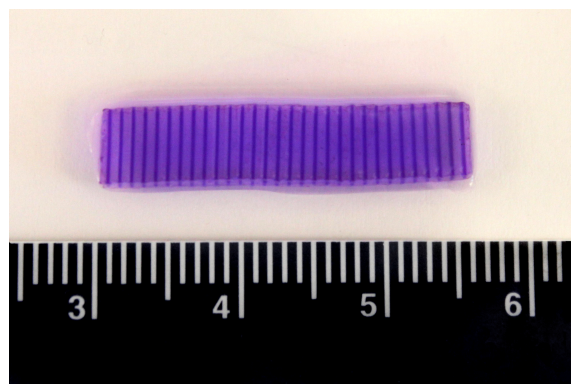

Fig. 1. Patterned PEGDA hydrogel sample (20\% pattern ratio), image courtesy of Dr. Xing Zhang, Rice University

\subsection{Base hydrogel and pattern stripe}

From the photolithographic patterning process, it can be seen that patterned PEGDA hydrogel samples are made of two different components, the base hydrogel and the pattern stripes. The base hydrogel contains $20 \mathrm{kDa}$ PEGDA molecules exclusively. On the other hand, the pattern stripes contain $20 \mathrm{kDa}$ PEGDA molecules and crosslinks formed between $20 \mathrm{kDa}$ and $3.4 \mathrm{kDa}$ molecules. In the finite element (FE) simulation, the base hydrogel is described with the modified neo-Hookean constitutive model

$$
\Psi_{m}(\boldsymbol{F})=\frac{1}{4} K\left(J^{2}-1-2 \ln J\right)+\frac{1}{2} G\left(\bar{I}_{1}-3\right)
$$


where $K$ is the bulk modulus, $G$ is the shear modulus, $J=\operatorname{det} \boldsymbol{F}$ is the determinant of the deformation gradient tensor $\boldsymbol{F}$, and $\bar{I}_{1}=J^{-\frac{2}{3}} \operatorname{tr}\left(\boldsymbol{F}^{\mathrm{T}} \boldsymbol{F}\right)$ is the modified tensor invariant. The volumetric term in Eq. 1 serves as a penalty to approximately capture the material incompressibility.

Pattern stripes are modeled as polymer networks embedded in the base hydrogel. This modeling choice enables us to capture the nonlinear relationship between the material stiffness and the pattern ratio of PEGDA hydrogels, which is demonstrated in the later part of this work. The fiber network generated by a random walk algorithm is used to represent the crosslinks formed between the $3.4 \mathrm{kDa}$ and $20 \mathrm{kDa}$ PEGDA molecules. The random walk algorithm has several control parameters. The fiber volume fraction ratio $\mu$ and the fiber diameter $d$ determine the density of the fiber network. Each fiber chain is piecewise linear and composed of multiple fiber segments with the length $l_{\text {seg }}$. The initial angle $\theta_{0}$ of individual fibers controls the network anisotropy. The relative angle $\theta_{r}$ between neighbouring segments follow the uniform distribution between $-\theta_{\max }$ and $\theta_{\max }$. The magnitude of $\theta_{\max }$ influences the network tortuosity. For the details of the random walk algorithm, interested reader is referred to [30].

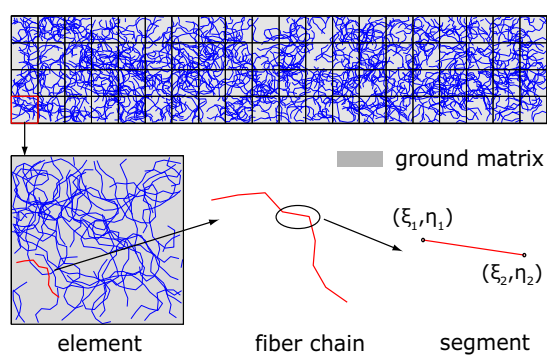

(a)

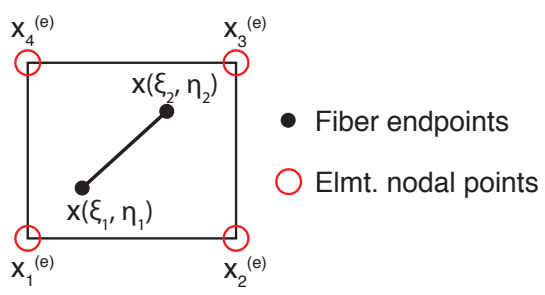

(b)

Fig. 2. (a) Pattern stripe meshed with four-node quad elements, the fiber network is generated by the random walk algorithm representing the crosslinks formed between $3.4 \mathrm{kDa}$ and $20 \mathrm{kDa}$ PEGDA molecules; (b) Projection of the DOFs of fiber segment to the DOFs of element nodes

Fig. 2a shows a pattern stripe meshed with four-node quad elements. Each finite element contains multiple piecewise linear fiber chains, and each fiber chain is composed of multiple segments. When a fiber segment crosses element 
boundaries, the coordinates of the intersections formed by the segment and the element boundaries are calculated, and the segment is divided into multiple sub-segments. The coordinates of the intersections between fiber segments and element boundaries, the crosslinks among different fiber chains, and the two endpoints of each fiber segment are collected in $\mathrm{L}=\left\{\left(x_{i}, y_{i}\right), i=1,2,3, \cdots\right\}$.

Inside an isoparametric element, the natural coordinates $\boldsymbol{\xi}$ and the Cartesian coordinates $\boldsymbol{x}$ have the following relationship:

$$
\boldsymbol{x}(\boldsymbol{\xi})=N_{a}(\boldsymbol{\xi}) \boldsymbol{x}_{a}^{(e)},
$$

where subscript $a$ is the element nodal number, $\boldsymbol{x}_{a}^{(e)}$ is the element nodal coordinates of the e-th element, and $N_{a}$ is the element interpolation function associated with node $a$. Based on Eq. 2, a mechanism illustrated in Fig. 2b is adopted to project the discrete degrees of freedom (DOFs) of fiber segments to the DOFs of element nodes. The natural coordinates $\boldsymbol{\xi}$ corresponding to the Cartesian coordinates of the fiber network recorded in L can also be obtained via the Newton-Raphson iterations.

The total strain energy of pattern stripes is expressed as follows

$$
\Pi(\boldsymbol{u})=\int_{\Omega_{0}} \Psi_{m}(\boldsymbol{F}(\boldsymbol{u})) \mathrm{d} \Omega+\sum_{i=1}^{N} \Psi_{f}\left(\lambda^{(i)}(\boldsymbol{u})\right),
$$

where $\boldsymbol{F}$ is the deformation gradient tensor, $\Psi_{m}$ is the strain energy function representing the contribution from the base hydrogel and takes the form of Eq. $1, \Psi_{f}$ is the fiber strain energy function representing the contribution from the $i$-th segment, $N$ is the total number of fiber segments contained in the fiber network, $\Omega_{0}$ is the reference domain, and $\lambda^{(i)}$ is the stretch ratio of the $i$-th fiber segment. After spatial discretization, the total strain energy becomes

$$
\begin{gathered}
\Pi(\boldsymbol{u})=\sum_{e=1}^{N_{e l}} \Pi^{(e)}(\boldsymbol{u})=\sum_{e=1}^{N_{e l}}\left(\Pi_{m}^{(e)}(\boldsymbol{u})+\Pi_{f}^{(e)}(\boldsymbol{u})\right), \\
\Pi_{m}^{(e)}(\boldsymbol{u})=\int_{\Omega_{0}^{(e)}} \Psi_{m}(\boldsymbol{F}(\boldsymbol{u})) \mathrm{d} \Omega, \quad \Pi_{f}^{(e)}(\boldsymbol{u})=\sum_{i=1}^{N^{(e)}} \Psi_{f}\left(\lambda^{(i)}(\boldsymbol{u})\right),
\end{gathered}
$$


where $N_{e l}$ is the total number of finite elements in the discretized system, $\Pi^{(e)}(\boldsymbol{u})$ is the strain energy of the $e$-th element and decoupled into two parts, the contributions from the base hydrogel $\Pi_{m}^{(e)}(\boldsymbol{u})$ and the embedded crosslinks formed between the $3.4 \mathrm{kDa}$ and $20 \mathrm{kDa}$ molecules $\Pi_{f}^{(e)}(\boldsymbol{u}), \Omega_{0}^{(e)}$ is the spatial domain occupied by the $e$-th element in the reference configuration, and $N^{(e)}$ is the number of fiber segments contained inside the $e$-th element.

The fiber segments are modeled as truss elements in the FE simulation with the assumption that fiber stretching is the main deformation mode [29, 27]. Since it is a widely accepted assumption that wavy fibers can only sustain tensile forces, but are not able to resist compression [31, 32, 33], the contribution from a fiber segment is switched off if its stretch ratio $\lambda<1$, indicating the compressive status. Considering the linear stress-strain relationship of the PEGDA hydrogels reported in the experiment [9], the fiber strain energy function $\Psi_{f}$ takes a polynomial form

$$
\Psi_{f}(\lambda)=\left\{\begin{array}{cl}
\frac{1}{2} k_{f} L_{0}^{2} \frac{(\lambda-1)^{2}}{\lambda^{z_{f}}} & \lambda \geq 1 \\
0 & 0<\lambda<1
\end{array}\right.
$$

where $k_{f}$ is the axial elastic modulus of fiber segment, $z_{f}$ is a dimensionless parameter controlling the fiber nonlinearity, $L_{0}$ is the segment original length, and $\lambda$ is the stretch ratio. It can be verified that $\left.\Psi_{f}(\lambda)\right|_{\lambda=1}=0$ and $\partial \Psi_{f}(\lambda) /\left.\partial \lambda\right|_{\lambda=1}=0$, indicating the continuity of the fiber strain energy and its first derivative at $\lambda=1$, and a stress-free state when no deformation occurs. Furthermore, the dimensionless parameter $z_{f}$ has to satisfy the constraint $z_{f} \leq 1$ to guarantee the convexity of the fiber strain energy function (Eq. 6). The detailed convexity analysis is shown in the Appendix.

For an arbitrary fiber segment contained in an element, $\boldsymbol{X}^{(1)}$ and $\boldsymbol{X}^{(2)}$ are used to represent the Cartesian coordinates of its two endpoints in the reference configuration. $\boldsymbol{x}^{(1)}$ and $\boldsymbol{x}^{(2)}$ are used to represent the corresponding coordinates in the deformed configuration. Based on Eq. 2, the stretch ratio of the fiber 
segment is expressed as follows:

$$
\lambda=\frac{l}{L_{0}}=\frac{\left\|\boldsymbol{x}^{(2)}-\boldsymbol{x}^{(1)}\right\|_{2}}{\left\|\boldsymbol{X}^{(2)}-\boldsymbol{X}^{(1)}\right\|_{2}}=\frac{\left\|N_{a}\left(\boldsymbol{\xi}^{(2)}\right) \boldsymbol{x}_{a}-N_{a}\left(\boldsymbol{\xi}^{(1)}\right) \boldsymbol{x}_{a}\right\|_{2}}{\left\|N_{A}\left(\boldsymbol{\xi}^{(2)}\right) \boldsymbol{X}_{A}-N_{A}\left(\boldsymbol{\xi}^{(1)}\right) \boldsymbol{X}_{A}\right\|_{2}} .
$$

170

Following a variational approach [30], the component forms of the element residual $\mathbf{R}_{f}^{(e)}$ and the stiffness matrix $\mathbf{K}_{f}^{(e)}$ contributed by a fiber segment under tension $(\lambda \geq 1.0)$ are derived:

$$
R_{f, i A}^{(e)}=k_{f} \frac{\lambda-1}{\lambda^{z_{f}+1}}\left(1-\frac{z_{f}}{2}+\frac{z_{f}}{2} \lambda^{-1}\right)\left(x_{i}^{(2)}-x_{i}^{(1)}\right)\left(N_{A}\left(\boldsymbol{\xi}^{(2)}\right)-N_{A}\left(\boldsymbol{\xi}^{(1)}\right)\right)
$$

and

$$
\begin{aligned}
K_{f, A i B j}^{(e)}= & k_{f} \frac{\lambda-1}{\lambda^{z_{f}+1}}\left(1-\frac{z_{f}}{2}+\frac{z_{f}}{2} \lambda^{-1}\right) \\
& \left(N_{A}\left(\boldsymbol{\xi}^{(2)}\right)-N_{A}\left(\boldsymbol{\xi}^{(1)}\right)\right)\left(N_{B}\left(\boldsymbol{\xi}^{(2)}\right)-N_{B}\left(\boldsymbol{\xi}^{(1)}\right)\right) \delta_{i j} \\
+ & \frac{k_{f}}{L_{0}^{2} \lambda^{z_{f}+2}}\left[\frac{z_{f}\left(z_{f}+2\right)}{2} \lambda^{-2}+\left(1-z_{f}^{2}\right) \lambda^{-1}+\frac{z_{f}\left(z_{f}-2\right)}{2}\right] \\
& {\left[\left(x_{i}^{(2)}-x_{i}^{(1)}\right)\left(N_{A}\left(\boldsymbol{\xi}^{(2)}\right)-N_{A}\left(\boldsymbol{\xi}^{(1)}\right)\right)\right] } \\
& {\left[\left(x_{j}^{(2)}-x_{j}^{(1)}\right)\left(N_{B}\left(\boldsymbol{\xi}^{(2)}\right)-N_{B}\left(\boldsymbol{\xi}^{(1)}\right)\right)\right] }
\end{aligned}
$$

where $A, B$ are the element nodal numbers, $i, j$ are the Cartesian directions, $x_{i}^{(1)}$ and $x_{i}^{(2)}, \boldsymbol{\xi}^{(1)}$ and $\boldsymbol{\xi}^{(2)}$ are the Cartesian and natural coordinates of the two endpoints of the $i$-th segment, and $N_{A}$ is the element interpolation function of node $A$.

The total element residual $\mathbf{R}^{(e)}$ and the stiffness matrix $\mathbf{K}^{(e)}$ are the sum of the contributions from the base hydrogel and the fiber network representing the crosslinks contained in the corresponding element

$$
\mathbf{R}^{(e)}=\mathbf{R}_{m}^{(e)}+\sum_{i} \mathbf{R}_{f_{i}}^{(e)}, \quad \mathbf{K}^{(e)}=\mathbf{K}_{m}^{(e)}+\sum_{i} \mathbf{K}_{f_{i}}^{(e)}
$$

Comparing the modeling strategies for the base hydrogel and the pattern, the strain energy of a finite element can be generalized as follows:

$$
\Pi^{(e)}(\boldsymbol{u})= \begin{cases}\Pi_{m}^{(e)}(\boldsymbol{u}) & N^{(e)}=0 \\ \Pi_{m}^{(e)}(\boldsymbol{u})+\Pi_{f}^{(e)}(\boldsymbol{u}) & N^{(e)}>0\end{cases}
$$


element. When $N^{(e)}=0$, the element is only composed of the base hydrogel represented by $\Pi_{m}^{(e)}(\boldsymbol{u})$. When $N^{(e)}>0$, the element is composed of the base hydrogel $\Pi_{m}^{(e)}(\boldsymbol{u})$ reinforced by the crosslink network, the contribution of which is represented by $\Pi_{f}^{(e)}(\boldsymbol{u})$.

The published experimental data from the uniaxial tension tests of the base hydrogel and $100 \%$ pattern [9] are used to calibrate the material parameters needed for the FE simulations. Fig. 3 shows the boundary conditions used in the $2 \mathrm{D}$ plane stress FE simulation that mimic the experimental setup.

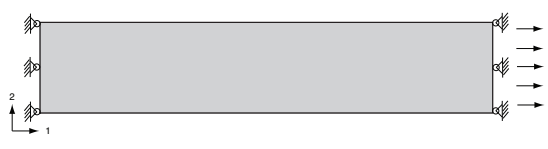

(a) Base hydrogel $(20 \mathrm{kDa})$

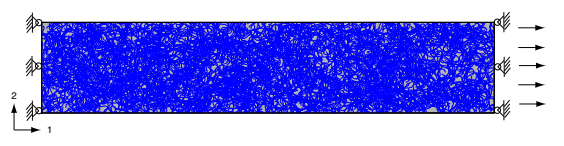

(b) $100 \%$ pattern $(3.4 \mathrm{kDa}+20 \mathrm{kDa})$

Fig. 3. Clamped-clamped boundary conditions are applied at the two ends of the hydrogel samples to mimic the setup of the uniaxial tension test

Recall that the base hydrogel is described by the modified neo-Hookean model (Eq. 1) with Poisson's ratio $\nu=0.49$ to approximately capture the material incompressibility. The only material parameter to determine is Young's modulus $E$, which is calibrated by solving the following optimization problem

$$
E=\underset{E}{\arg \min } \sum_{i=1}^{m}\left\|P_{11}\left(E, \lambda^{(i)}\right)-P_{11}^{*}\left(\lambda^{(i)}\right)\right\|_{2},
$$

where $P_{11}$ is the nominal stress (force divided by the sample cross section area in the initial configuration) in the loading direction obtained from the FE simulation, $P_{11}^{*}$ is the counterpart obtained from the experiment, $\lambda$ is the sample stretch ratio (strain), and $m$ is the number of available experimental data pairs. Unless otherwise specified, in the following stress refers to the nominal stress and strain refers to the sample stretch ratio.

The $100 \%$ pattern sample is modeled as a fiber network (representing the crosslinks formed between $20 \mathrm{kDa}$ and $3.4 \mathrm{kDa}$ molecules) embedded in the 
base hydrogel. According to Eq. 4, the total strain energy of the $100 \%$ pattern sample is decoupled into the contributions from the base hydrogel and the crosslinks formed between $3.4 \mathrm{kDa}$ and $20 \mathrm{kDa}$ molecules. Therefore, the Young's modulus $E$ of the base component in the pattern stripe takes the same value as calibrated according to Eq. 12 . Fig. 3b shows a $100 \%$ pattern sample with an embedded fiber network synthetically generated via the 2D random walk algorithm. Since the parameters of the crosslinks are not available, part of the parameter values are chosen from the range reported in the literature. For example, the fiber diameter $d=0.45 \mathrm{~nm}$ [20]. The crosslink network in the pattern sample is isotropic, therefore all the fiber chains are evenly distributed in different orientations without a preferred direction, $\theta_{0} \sim \operatorname{unif}\left(0^{\circ}, 360^{\circ}\right)$. The relative angle between neighbouring segments follows a uniform distribution, $\theta_{r} \sim \operatorname{unif}\left(-90^{\circ}, 90^{\circ}\right)$. The fiber volume fraction ratio (crosslink density) $\mu$, the

segment length $l_{\text {seg }}$, the fiber axial stiffness matrix $k_{f}$, and the fiber nonlinear parameter $z_{f}$ are treated as an ensemble and calibrated by solving the following optimization problem

$$
\left(\mu, l_{\text {seg }}, k_{f}, z_{f}\right)=\underset{\left(\mu, l_{\text {seg }}, k_{f}, z_{f}\right)}{\arg \min } \sum_{i=1}^{m}\left\|P_{11}\left(\mu, l_{\text {seg }}, k_{f}, z_{f}, \lambda^{(i)}\right)-P_{11}^{*}\left(\lambda^{(i)}\right)\right\|_{2},
$$

190 and the experiment of the $100 \%$ pattern sample, respectively.

\subsection{Pattern topology}

The mechanical behavior of patterned PEGDA hydrogel largely depends on the pattern topology. The FE simulation result of the hydrogel sample with $20 \%$ pattern ratio is firstly compared with the experimental data reported in [9]. Then, a thorough numerical investigation is performed to examine the influence of different pattern geometries, including pattern ratio, orientation and waviness, on the material mechanical behavior. Through this process, the nonlinear relationship between the pattern ratio and the material linear stiffness, which is an important phenomenon reported in the literature, is recovered. Furthermore, the three-region (toe-heel-linear) stress-strain relationship that is 
typically exhibited by biological tissues is also captured by tuning the hydrogel property.

\subsubsection{Pattern ratio}

During the photolithographic patterning process, the pattern ratio of hydrogel samples can be tuned according to the pre-designed photomask. In the experiment [9], PEGDA hydrogel samples with parallel pattern stripes were synthesized. The width of each pattern stripe was $100 \mu \mathrm{m}$, and the width of the base hydrogel between two neighbouring pattern stripes was $400 \mu \mathrm{m}$. Therefore, the pattern ratio of the sample was $20 \%$. In the uniaxial tension test, the hydrogel samples with $20 \%$ pattern ratio were stretched along the parallel and perpendicular directions with respect to the stripe orientation, respectively. Fig. 4 shows the boundary conditions used in the FE simulation mimicking the setup of the experiments. The crosslink network contained in the pattern stripes are generated via the random walk algorithm. The material parameters calibrated from the base hydrogel and the $100 \%$ pattern according to Eqs. 12 and 13 directly used in the FE simulation, the numerical results obtained from which are compared with the experimental data.

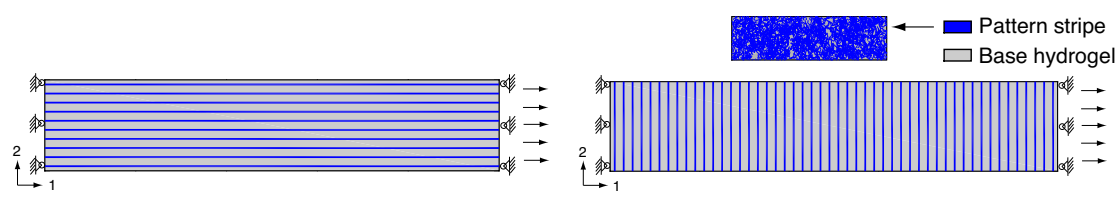

(a) Parallel loading

(b) Perpendicular loading

Fig. 4. Boundary conditions used in the FE simulations to mimic the setup of the uniaxial tenion tests of hydrogel samples with $20 \%$ pattern ratio

Through similar approaches, hydrogel samples with other pattern ratios $(40 \%, 60 \%$ and $80 \%)$ are generated via the random walk algorithm, and their mechanical behavior is numerically investigated to quantify the influence of pattern ratio. 


\subsubsection{Pattern orientation}

In the uniaxial tension tests of the hydrogel sample with $20 \%$ pattern ratio, the material parameters for the base hydrogel and the pattern stripes take the values calibrated according to Eqs. 12 and 13.

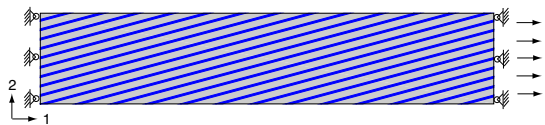

(a) $\theta=15^{\circ}$

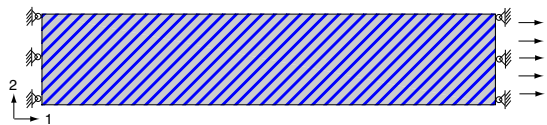

(b) $\theta=45^{\circ}$

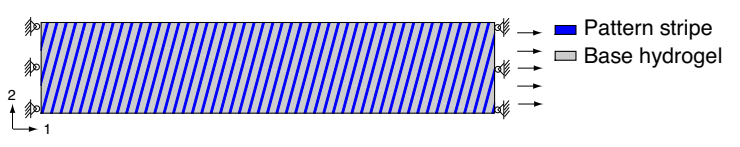

(c) $\theta=75^{\circ}$

Fig. 5. Pattern stripes form different angle $\theta$ with respect to the tensile loading

\subsubsection{Pattern waviness} parallel to each other. With the help of the numerical strategy, the influence of more complex pattern geometries can be explored in FE simulations. For example, wavy patterns can be adopted to tune the material mechanical behavior. A rectangular PEGDA hydrogel sample patterned with a single sinusoidalclamped-clamped boundary conditions are used. The hydrogel sample has the dimensions of $25 \times 5 \mathrm{~mm}$ and undergoes displacement controlled uniaxial ten- 
sion. The influence of the pattern waviness, characterized by the ratio between the amplitude $A$ and the half wavelength $L$ of the sinusoidal pattern stripe, is quantified numerically. We investigate the influence of $A / L$ following two approaches. First, the half wavelength $L$ is fixed and the amplitude $A$ varies. This approach generates the same number of half waves $N$ in the sample (Fig. 6). Then, the amplitude $A$ is fixed and the half wavelength $L$ varies, generating different number of half waves $N$ in the sample (Fig. 7).

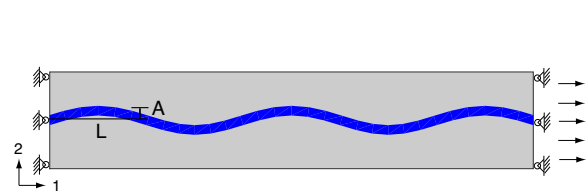

(a) $A / L=0.1, N=5$

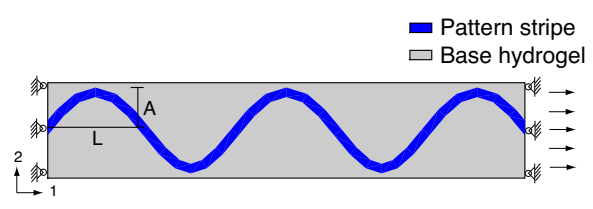

(b) $A / L=0.4, N=5$

Fig. 6. Hydrogel sample with a sinusoidal stripe under uniaxial tension, the half wavelength $L$ is fixed and the amplitude $A$ varies

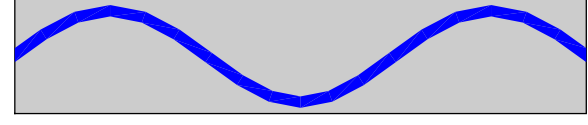

(a) $A / L=0.24, N=3$

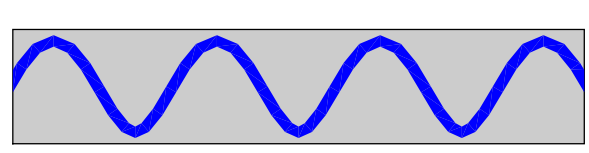

(c) $A / L=0.56, N=7$

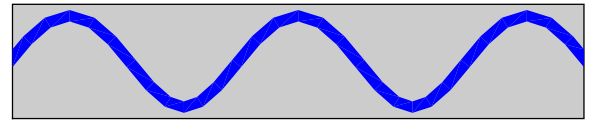

(b) $A / L=0.40, N=5$

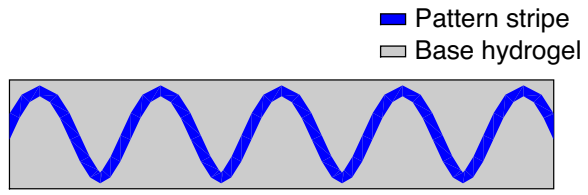

(d) $A / L=0.72, N=9$

Fig. 7. Hydrogel sample with a sinusoidal stripe under uniaxial tension, the amplitude $A$ is fixed and the half wavelength $L$ varies

One of the design objectives is to ensure patterned PEGDA hydrogels possess a stress-strain relationship similar to the one typically exhibited by native biological tissues, as shown in Fig. 8. The shape of the stress-strain curve of the patterned PEGDA hydrogel shown in Figs. 6 and 7 mainly depends on two 
factors, the relative stiffness between the pattern stripe and the base hydrogel, and the pattern stripe waviness. Recall that the material parameters of the pattern stripe $\left(\mu, l_{\text {seg }}, k_{f}, z_{f}\right)$ are calibrated as an ensemble, where $\mu$ represents the crosslink density. Generally speaking, the pattern stiffness increases as the crosslink density $\mu$ increases. In the $\mathrm{FE}$ simulation, by means of tuning the value of $\mu$ while keeping the other parameters constant, the influence of the relative stiffness between the pattern stripe and the base hydrogel on the shape of the stress-strain curve of patterned PEGDA hydrogels is investigated.

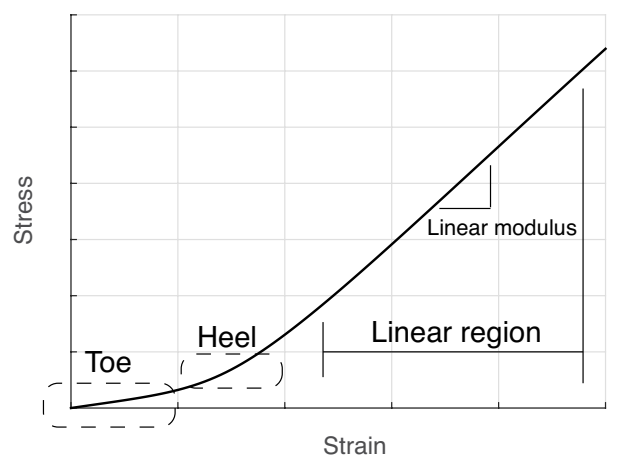

Fig. 8. A typical stress-strain curve of biological tissues can be divided into three regions, a toe region followed by a heel region and a linear region

\section{Results}

The generation of different geometric patterns in the hydrogel samples is based on the random walk algorithm and automated in MATLAB ${ }^{\circledR}$. According to the component forms of the element residual and stiffness matrix shown in Eqs. 8 and 9, a user element subroutine is implemented in an open source finite element analysis package FEAP [34].

In the random walk algorithm used for generating the fiber network, the initial angle $\theta_{0}$ of each fiber chain and the relative angle $\theta_{r}$ between two neighboring fiber segments are random variables and follow two uniform distributions, respectively. The starting point of each fiber chain is also random. As a result, even with the same values of the algorithmic parameters, the generated fiber 
network varies between identical simulations. To consider the influence of the stochastic nature of the network on the numerical results, the Monte Carlo (MC) method is combined with FE simulations to capture the material average behavior. In this research, we focus on the material stress-strain relationship and the following MC approach is adopted:

for $i=1: N$ do

Random walk algorithm;

Finite Element simulation;

Mean stress $\bar{\sigma}=\sum_{j=1}^{i} \sigma(j) / i$;

end for

std $=\sqrt{\frac{\sum_{i=1}^{N}(\sigma-\bar{\sigma})^{2}}{N}}$

where $N$ is the number of MC simulations, $\bar{\sigma}$ and std are the mean stress and the standard deviation corresponding to a specific strain level.

In the following FE simulations, hydrogel samples undergoing uniaxial tension are modeled as 2D plane stress problems with clamped-clamped boundary conditions to mimic the experiment setup. All the samples have the dimensions of $25 \times 5 \mathrm{~mm}$, the same as used in the experiment, unless otherwise specified. 290 Four-node quadrilateral elements are used. $N=500 \mathrm{MC}$ simulations are performed to obtain the mean and standard deviation of the sample stress-strain relationship. One of the advantages of using the element formulation adopted (Eq. 11) is that for hydrogels with complex geometric patterns, structured FE meshes can still be used. This is due to the fact that the contribution from the crosslink network is directly considered at the element level. For example, Fig. 9 shows a hydrogel sample with a sinusoidal pattern stripe meshed by four-node quadrilateral elements. The crosslink network in the pattern stripe is generated via the random walk algorithm. The $i$ th element only contains the base hydrogel, therefore $N^{(i)}=0$ (recall that $N^{(i)}$ represents the number of 300 segments contained in that element) and the element is described by the modified neo-Hookean model. On the other hand, the $j$ th element contains part of the pattern stripe, therefore $N^{(j)}>0$ and the element is described by the 
modified neo-Hookean model enhanced by the contribution from that part of the crosslink network.

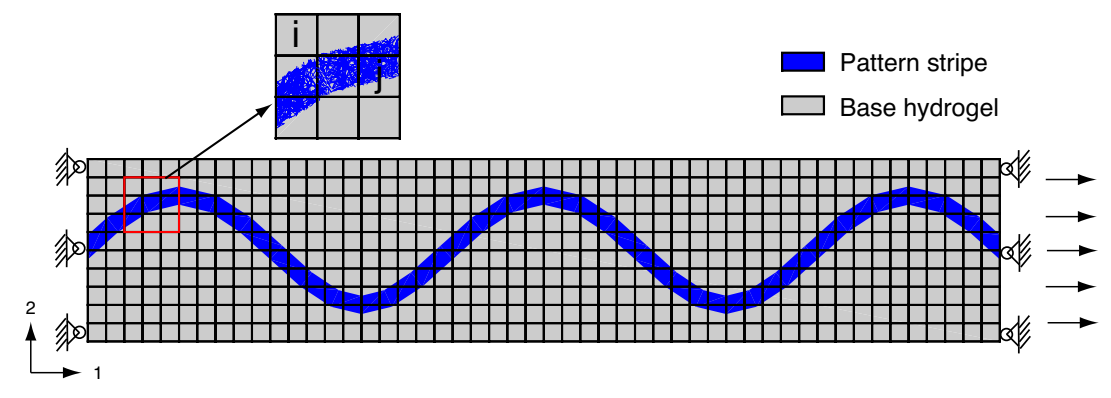

Fig. 9. Hydrogel sample with a sinusoidal pattern stripe meshed by four-node quadrilateral elements

Table 1 shows the material parameters of the base hydrogel and the $100 \%$ pattern sample calibrated from the experimental data, according to Eqs. 12 and 13. Recall that the base hydrogel is described by the neo-Hookean model, containing two material parameters Young's modulus $E$ and Poisson's ratio $\nu$. The pattern is composed of the base hydrogel enhanced by the crosslinks formed between $20 \mathrm{kDa}$ and $3.4 \mathrm{kDa}$ molecules. The Young's modulus $E$ and Poisson's ratio $\nu$ used in the pattern represent the mechanical properties of its base component and take the same values as the base hydrogel. The material parameters $\mu, l_{\text {seg }}, k_{f}, z_{f}$ used in the pattern represent the mechanical properties of its embedded fiber network. Fig. 10 shows the comparison of the stress-strain curves obtained from the FE simulations and the experimental data of the base hydrogel and $100 \%$ pattern samples under uniaxial tension, where $N=500$ $\mathrm{MC}$ simulations are used to obtain the mean and the standard deviation of the stress-strain relationship.

Comparing Figs. 10a and 10b it can be seen that the pattern sample is much 320 stiffer than the base hydrogel due to the crosslink network between $3.4 \mathrm{kDa}$ and $20 \mathrm{kDa}$ in the former. Note that the material parameters of the crosslink network $\left(\mu, l_{\text {seg }}, k_{f}, z_{f}\right)$ are calibrated here as an ensemble, and the optimization problem (Eq. 13) typically has multiple local minima. Consequently, the value 
Table 1. Material parameters of the base hydrogel and the $100 \%$ pattern sample calibrated from uniaxial tension tests

\begin{tabular}{c|cccccc}
\hline Parameters & $E(\mathrm{kPa})$ & $\nu$ & $\mu$ & $l_{\text {seg }}(\mu \mathrm{m})$ & $k_{f}\left(\mathrm{Nmm}^{-1}\right)$ & $z_{f}$ \\
\hline Base hydrogel & 5.5 & 0.49 & - & - & - & - \\
\hline $100 \%$ pattern & 5.5 & 0.49 & 0.01 & 75 & 0.04 & 0.4 \\
\hline
\end{tabular}

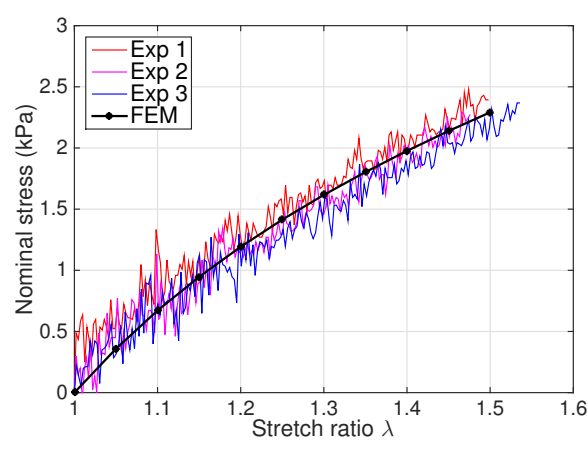

(a) Base hydrogel

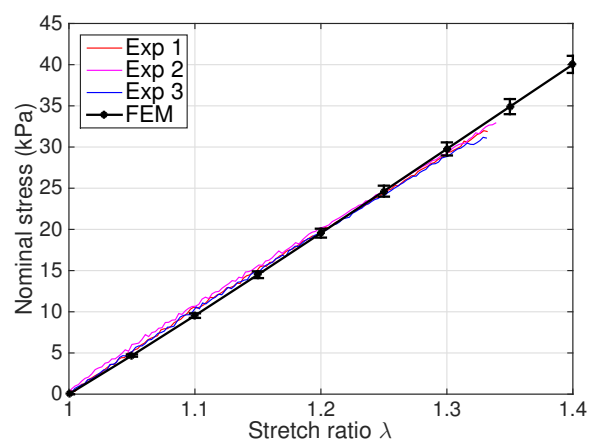

(b) $100 \%$ pattern

Fig. 10. Calibration: comparison of stress-strain relationships obtained from simulations (FEM) and experiments (Exp 1,2,3), experimental data courtesy of Dr. Xing Zhang and Dr. Jane Grande-Allen, Rice University [9]

of individual parameters in the ensemble $\left(\mu, l_{s e g}, k_{f}, z_{f}\right)$ may not be uniquely determined. That is to say, there may exist multiple combinations of crosslink network parameters that fit the experimental data shown in Fig. 10b equally well, and the group listed in Table 1 is only one of them. However, since these material parameters all have straightforward physical or geometric meaning, they can ideally be measured from experiments. The current parameter fitting process is only a compromising approach due to lack of detailed information about the microscopic structure of the fabricated material. In the following FE simulations, the material parameters listed in Table 1 are used for the base hydrogel and the pattern stripes of PEGDA hydrogels with different pattern geometries. 
In the experiment carried out in [9], the hydrogel samples with $20 \%$ pattern ratio were stretched along the direction that was either parallel or perpendicular to the pattern stripes. Since pattern stripes are much stiffer than the base hydrogel, patterned PEGDA hydrogels are anisotropic, with higher stiffness along the pattern orientation. Fig. 11 shows the comparison of the stress-strain curves obtained from the FE simulations and the experiments of the hydrogel samples with $20 \%$ pattern ratio under parallel or perpendicular tensile loading, where $N=500 \mathrm{MC}$ simulations are used. The numerical results match the experimental data well in both cases.

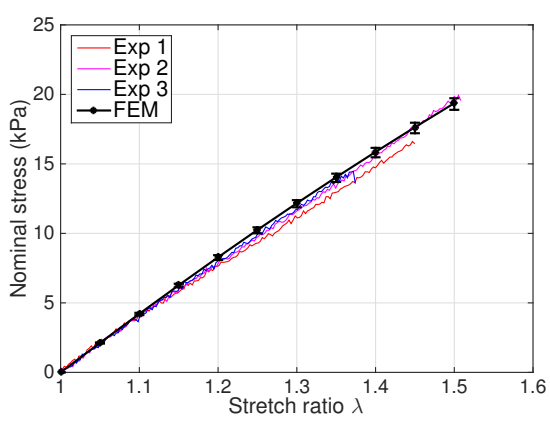

(a) Parallel loading

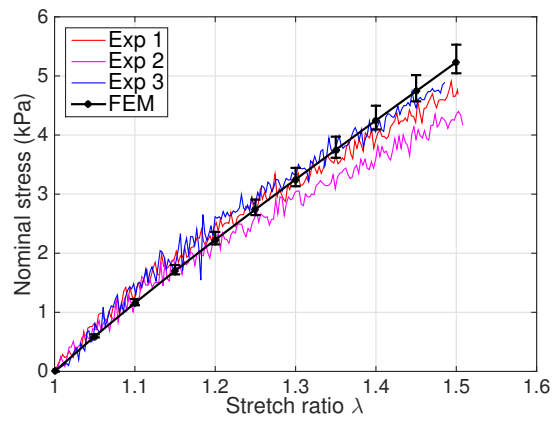

(b) Perpendicular loading

Fig. 11. Validation: comparison of stress-strain relationships obtained from simulations (FEM) and experiments (Exp 1,2,3) of hydrogel samples with $20 \%$ pattern ratio, experimental data courtesy of Dr. Xing Zhang and Dr. Jane Grande-Allen, Rice University [9]

The influence of the pattern ratio on the mechanical behavior of PEGDA hydrogel is further numerically investigated. The sample dimensions and the boundary conditions are kept the same as the counterparts used in the case for the $20 \%$ pattern ratio. The relationship between the pattern ratio and linear modulus (the slope of the linear portion of the stress-strain curve) of patterned PEGDA hydrogels is shown in Fig. 12. For the 0\% (base hydrogel), 20\% and $100 \%$ pattern ratios, the FE results match the experimental data well in both the parallel and perpendicular loading cases. Scrutinizing the experimental data, it can be seen that the linear modulus $(100 \mathrm{kPa})$ of the $100 \%$ pattern sample 
is 20 times larger than the value $(4.5 \mathrm{kPa})$ of the base hydrogel $(0 \%)$. When the pattern ratio is increased from $0 \%$ to $20 \%$, the material linear modulus is increased from $4.5 \mathrm{kPa}$ to $40 \mathrm{kPa}$, which is $40 \%$ of the $100 \%$ pattern sample (Fig. 12a). When the pattern ratio is increased to $80 \%$, the linear modulus predicted by the FE simulation is almost as large as the value of the $100 \%$ pattern sample, indicating a saturation state for the crosslink network formed between $3.4 \mathrm{kDa}$ and $20 \mathrm{kDa}$ PEGDA molecules.

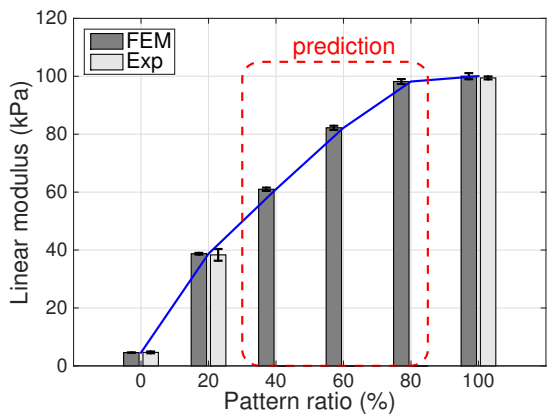

(a) Parallel loading

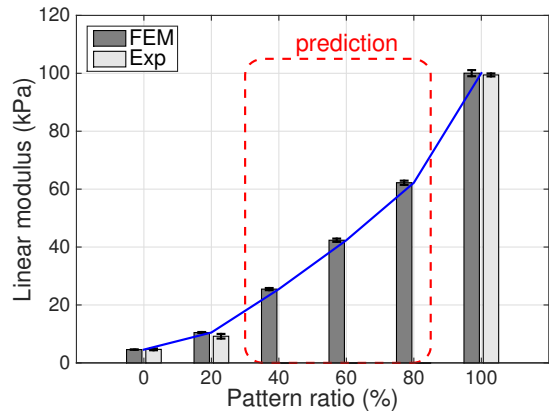

(b) Perpendicular loading

Fig. 12. Relationship between pattern ratio and linear moduli of patterned hydrogels patterned PEGDA hydrogels predicted by FE simulations. When the pattern ratio is $20 \%$, the ratio between the material linear moduli for the parallel loading and the perpendicular loading is around 3.8. When the pattern ratio is increased to $80 \%$, the ratio decreases to around 1.5 , indicating that the material anisotropy becomes less obvious.

The nonlinear relationship between the pattern ratio and the material linear modulus, the saturation phenomenon of the crosslinking, and the decrease of the material anisotropy with the increase of the pattern ratio, were all reported in the literature, see $[16,9]$. The above phenomena are all successfully captured 370 in the FE simulations, demonstrating the capabilities of the adopted numerical strategy.

In the uniaxial tension test of the hydrogel sample with $20 \%$ pattern ratio, 


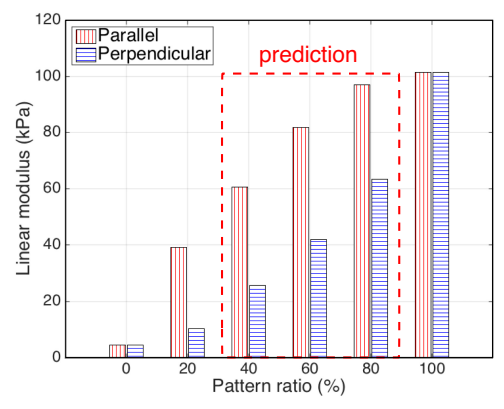

(a)

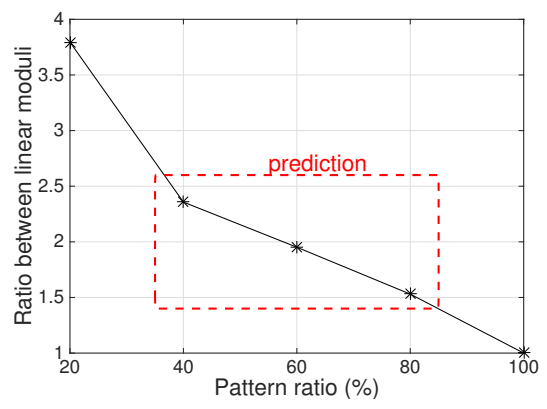

(b)

Fig. 13. (a) Relationship between pattern ratio and material anisotropy of patterned hydrogels; (b) Ratio between material linear moduli in the parallel and perpendicular directions

the pattern stripes are either parallel or perpendicular with respect to the loading direction. The influence of other pattern orientations $(\theta)$ are also examined numerically. Fig. 14 shows the relationship between the pattern orientation and the linear modulus of the hydrogel sample with $20 \%$ pattern ratio. The linear moduli predicted by the FE simulations decrease as the angle $\theta$ formed between the pattern stripes and the tensile loading direction increases, indicating that the adopted numerical strategy is capable to capture the material anisotropy.

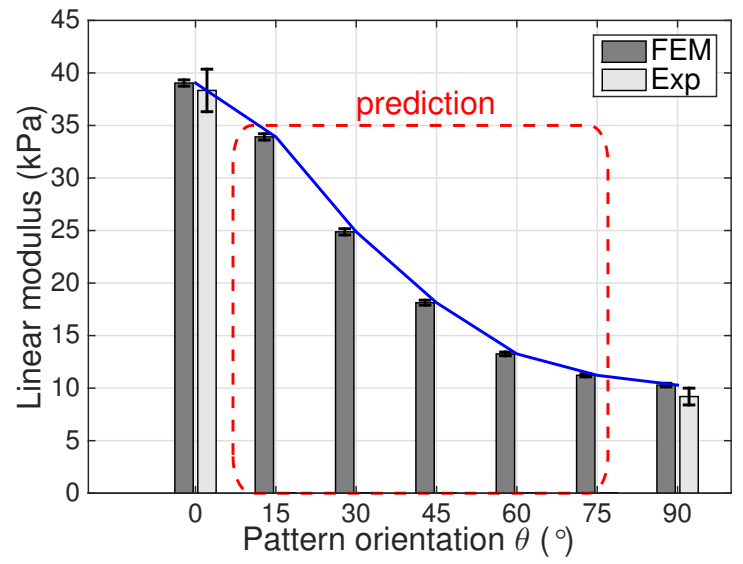

Fig. 14. Relationship between pattern orientation $\theta$ and linear modulus of the hydrogel sample with $20 \%$ pattern ratio 
Recall that for the hydrogel patterned with a sinusoidal stripe, which is generated via the random walk algorithm, the pattern waviness is characterized by the ratio between the amplitude $A$ and the half wavelength $L$ of the sinusoidal pattern stripe (Fig. 6). In the FE simulation, the material parameters used for the base hydrogel and the sinusoidal pattern stripe are from the values listed in Table 1. Since the standard deviation is negligible compared with the mean in the MC simulations, only the mean stress-strain relationships are reported from now on. From the simulation results shown in Fig. 15, it can be seen that the sample linear modulus decreases when the pattern stripe becomes wavier $(A / L$ increases), although the sample with a wavier stripe has a larger pattern ratio.

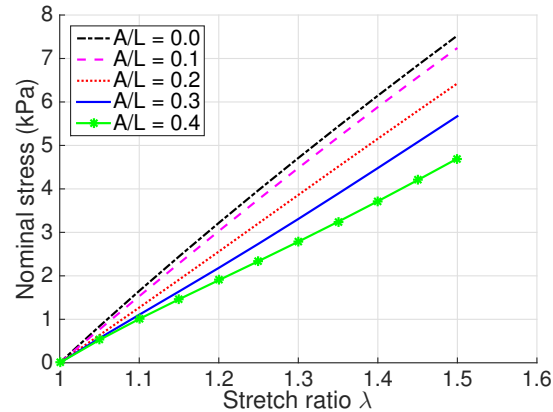

(a) Stress-strain relationship

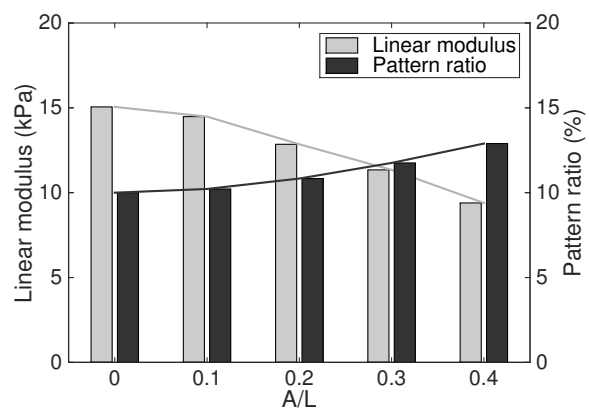

(b) Linear modulus

Fig. 15. Prediction: relationship between the pattern waviness $(A / L)$ and the linear modulus of the hydrogel sample patterned with one sinusoidal stripe

As shown in Fig. 15, although the hydrogel sample is patterned with a sinusoidal stripe, the stress-strain curve obtained from the FE simulation is still linear. In order to acquire the three-region (toe-heel-linear) stress-strain relationship typically exhibited by biological tissues, the relative stiffness of the sinusoidal pattern stripe with respect to the base hydrogel is tuned by increasing the crosslink density $\mu$ in the simulation. Fig. 16 shows the relationship between the crosslink density $\mu$ and the linear modulus of the pattern stripe normalized with respect to the base hydrogel. The linear relationship between $\mu$ and the pattern stripe stiffness is a manifestation of the underlying affine fiber kinematics 
$[35,36,37,38]$ of the adopted numerical strategy.

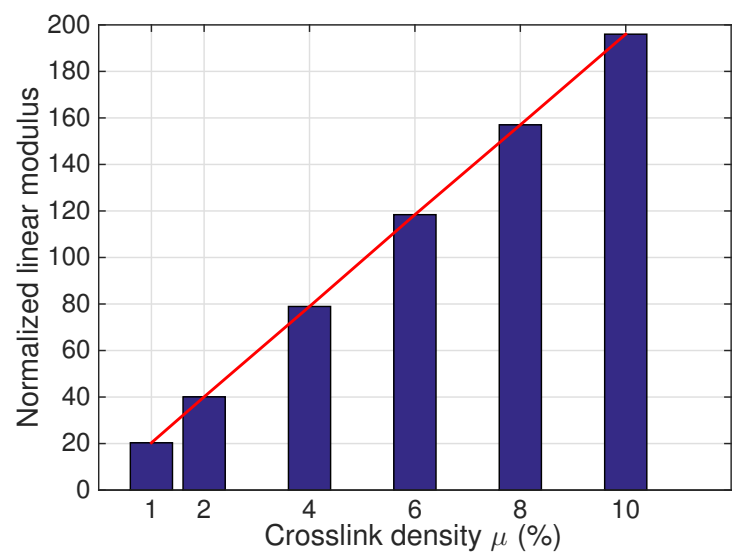

Fig. 16. Prediction: relationship between the crosslink density $\mu$ and the linear modulus of pattern stripe normalized with respect to the base hydrogel

In the following $\mathrm{FE}$ simulations, the value of the crosslink density $\mu$ in the pattern stripe is increased from 0.01 to 0.10 , and the rest of the material parameters shown in Table 1 are kept the same. Fig. 17a shows the influence of $\mu$ on the stress-strain relationship of the hydrogel sample patterned with a sinusoidal-shape stripe (Fig. 6), where $A / L=0.4$. It can be seen that the slope and the length of the toe region on the stress-strain curves are not influenced by the crosslink density $\mu$. This is because when the sample stretch is small $(\lambda<1.1)$, most crosslink fibers are still in the crimp state and do not contribute to the material stiffness. Therefore, the material global behavior is not influenced by the amount of fibers contained in the material (the crosslink density) at this stage. However, the slope of the linear region on the stress-strain curve increases as the value of $\mu$ increases. This is because when the stretch is large enough $(\lambda>1.3)$, most crosslink fibers are straightened, and the material stiffness is directly proportional to the crosslink density $\mu$. Fig. 17b shows the stress-strain curves normalized with respect to the stress when the sample stretch ratio $\lambda=2.0$. It can be seen that the three-region (toe-heel-linear) characteristic of the stress-strain curve becomes more obvious when the crosslink density $\mu$ (or the relative stiffness of the pattern stripe with respect to the base 
hydrogel) increases.

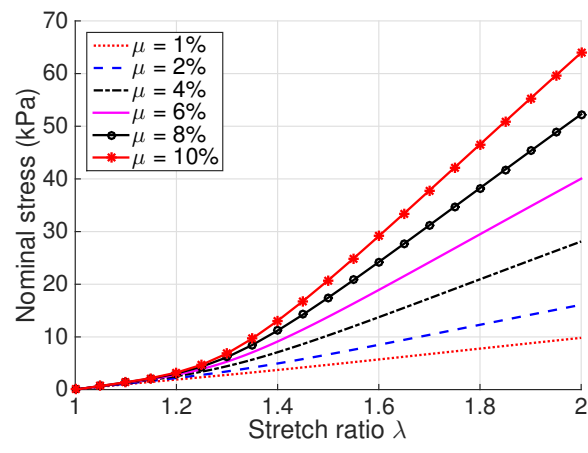

(a) Influence of the crosslink density $\mu$

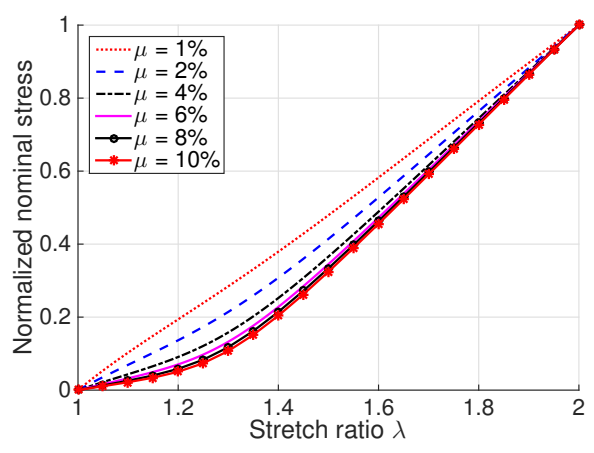

(b) Normalized stress-strain relationships

Fig. 17. Prediction: influence of the crosslink density $\mu$ on the stress-strain relationship of the hydrogel sample patterned with a sinusoidal stripe, $A / L=0.4$

Fig. 18 shows how the change of the pattern waviness relates to different regions on the nonlinear stress-strain curve. When the stretch ratio $\lambda$ is small (from 1.0 to 1.2), the stress-strain curve is in the toe-region due to the large waviness of the pattern stripe. The heel region approximately begins at $\lambda=1.2$, marked by point $(b)$, and ends at $\lambda=1.4$, marked by point $(c)$ on the curve. After the exit of the heel region, the stripe waviness decreases significantly compared with the initial stage, contributing to a larger material modulus in the linear region. Fundamentally, the increase of the material stiffness is triggered by two mechanisms, the straightening of individual fibers at the microscale, and the straightening of the pattern stripe at the macroscale.

The shape of the three-region stress-strain curve can be further tuned by adjusting the number of half waves $N$ of the sinusoidal pattern stripe (Fig. 7). Fig. 19 shows the influence of the number of half waves $N$ ( $A$ is fixed and $L$ varies) on the length of the toe region and the slope of the linear region obtained from the FE simulation. In Fig. 19b, the toe-region length is normalized to the total sample stretch ratio $\lambda=2.0$. It can be seen that when $N$ increases, the length of the toe region increases, and the slope of the linear region after the upward heel decreases. 

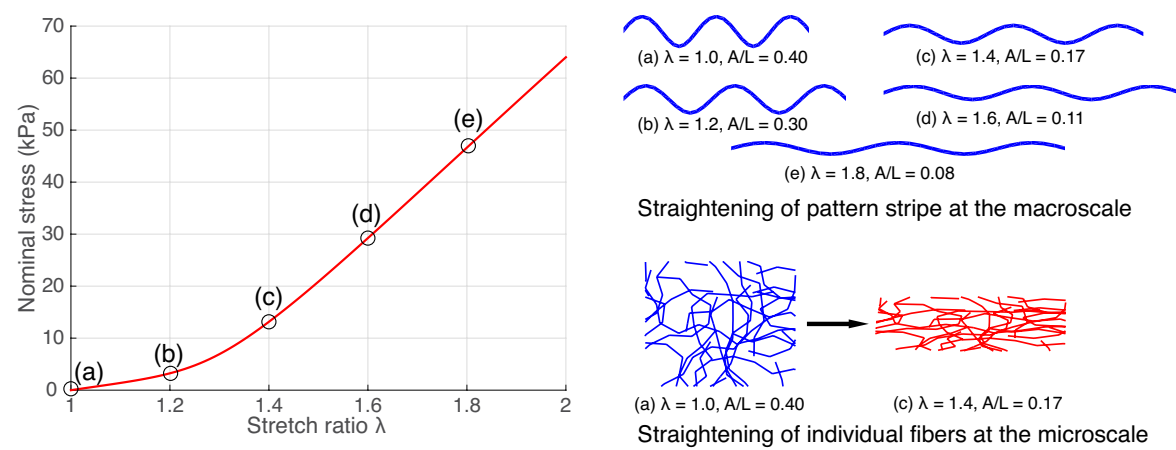

Fig. 18. Straightening of pattern stripe at the macroscale and individual fibers at the microscale

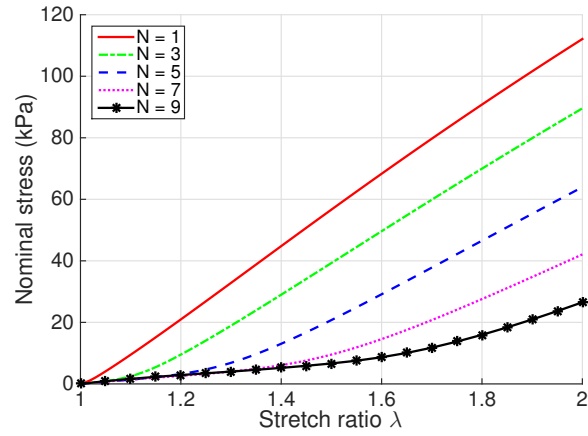

(a) Stress-strain curves

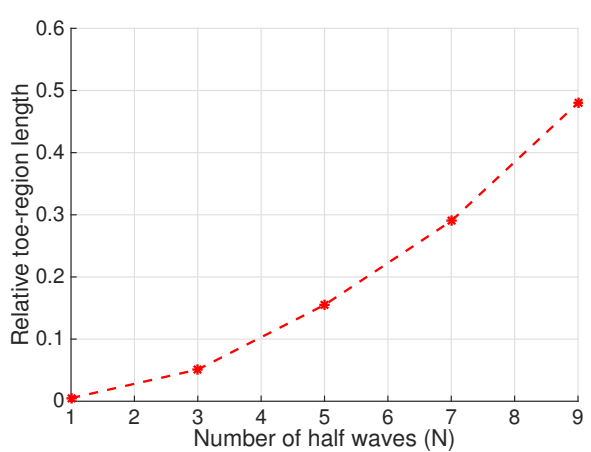

(b) Toe-region length

Fig. 19. Prediction: influence of the number of half waves $N$ of the sinusoidal pattern stripe on the stress-strain relationship

The material parameters of the base hydrogel and the pattern stripe(s) used in the above FE simulations are directly calibrated from the experiment and listed in Table 1. From the experimental data shown in Fig. 10, we can see that the pattern stripe behaves linearly under uniaxial tension. Therefore, a wavy (sinusoidal) shape of stripe is necessary to ensure that the patterned hydrogel sample possesses a toe-heel-linear stress-strain relationship, as shown in Figs. 17 and 18 . However, by properly tuning the mechanical properties of the fiber component in the pattern stripe, the FE simulation predicts that hydrogel samples 


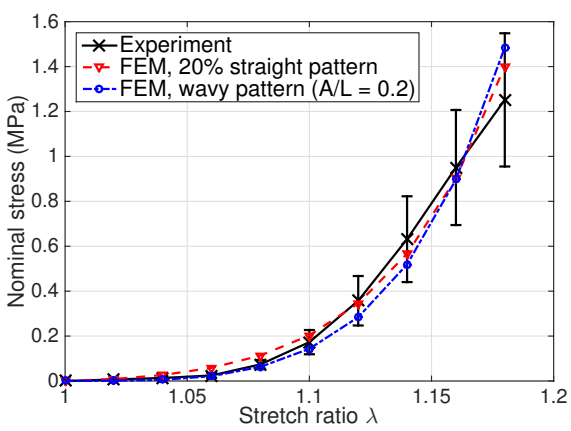

(a) Circumferential direction

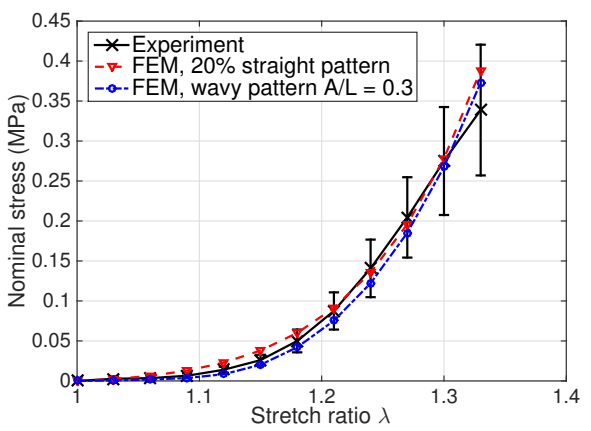

(b) Radial direction

Fig. 20. Comparison between the FE simulations and the experimental data of porcine aortic valve tissues, experimental data are obtained from [39] 


\section{Discussions}

Among many potential biomedical applications, patterned PEGDA hydrogels can be used as scaffold materials to fabricate tissue engineered heart valves

465 pend on the pattern topology. In this paper, we adopt a newly proposed computational framework [30] combined with published experimental data [9] to numerically quantify the influence of various pattern topology, including pattern ratio, orientation and waviness, on the mechanical behavior of PEGDA 
elastic modulus $k_{f}$ and the dimensionless parameter $z_{f}$ of individual fibers. The material parameter calibration process for the pattern is more complex in the sense that there may exist multiple groups of parameter values of $\left(\mu, l_{s e g}, k_{f}, z_{f}\right)$ fitting the experimental data equally well. Fitting these parameters as an ensemble is only a compromising approach adopted in this paper, due to lack of the quantitative information about the microscopic structure of the pattern. However, recently many efforts have been devoted to study the mechanical behavior of a single fiber $[40,41,42]$. Also, quantitative geometric information regarding microscopic fiber arrangement can be acquired via advanced image processing technologies such as confocal reflection imaging [20,43] or scanning electron microscopy (SEM) [25]. All these advances make it possible to uniquely determine the material parameters of the crosslink network from experiments.

The nonlinear relationship between the pattern ratio and the linear modulus of patterned PEGDA hydrogels was reported by different researchers in the literature $[16,9]$. This phenomenon is successfully captured by the numerical strategy adopted in this paper. At the same time, the decrease of the material anisotropy when the pattern ratio increases, which was observed in the experiment [16], is also captured in the FE simulation. Currently, only the numerical results of hydrogel samples with $0 \%, 20 \%$ and $100 \%$ pattern ratios are compared with the experimental data (Fig. 12). To further verify the nonlinear relationship between the pattern ratio and the material linear modulus obtained from the numerical investigation, more experiments (hydrogel samples with other pattern ratio) are still needed.

In the context of TEHVs, patterned PEGDA hydrogels are designed to mimic the mechanical behavior of native heart valve tissues, so that the valve can provide sufficient mechanical functions after initial implantation, which is crucial for the survival of the patient [4]. The numerical results presented in this paper predict that patterned hydrogels can be designed via two approaches to 520 possess a toe-heel-linear stress-strain relationship exhibited by many native biological tissues. When pattern stripes behave according to a linear stress-strain relationship (Fig. 10b), as reported in the experiment by [9], wavy patterns 
are necessary to ensure the hydrogel sample possess a three-region stress-strain curve. An alternative approach is to increase the nonlinearity of the fiber component contained in pattern stripes. Following this approach, the FE simulation demonstrates that hydrogel samples with straight patterns can also exhibit a nonlinear stress-strain relationship (Fig. 20). In general, there may exist different combinations of material properties and pattern geometries to get a similar stress-strain relationship. In the actual pattern design process, which pattern geometry to be adopted largely depends on the targeted stress-strain relationship and the material properties of the base and the pattern. Furthermore, during the fabrication and patterning process of hydrogels, there are many constraints on the base and the pattern properties. For example, only certain range of crosslinking density $\mu$ or fiber stiffness $k_{f}$ could be reached. In order to de535 sign a patterned hydrogel satisfying certain targeted mechanical properties, the constraints on the material properties also need to be considered, making the whole design process become a constrained optimization problem.

The relative stiffness between the pattern stripe and the base hydrogel influences the stress-strain relationship of patterned hydrogels. The FE simulation suggests that the toe-heel-linear behavior occurs when the stripe is much stiffer than the base hydrogel. This observation is supported by the comparison of material properties of extracellular matrix (ECM) and collagen fibers reported in literature. The mechanical properties of several tissue-specific ECM measured by atomic force microscopy are summarized in [44]. For example, the elasticity of ECM in smooth muscle is from 5 to $9 \mathrm{kPa}$ [45], and the elasticity of ECM in skeletal muscle varies from 8 to $17 \mathrm{kPa}[46,47]$. The elastic moduli of single collagen fibers reported in the literature are rather scattered. For example, the elastic moduli for single fibers extracted from different rat tails are reported to be between $100 \mathrm{MPa}$ and $360 \mathrm{MPa}$ [48]. The (tangent) elastic moduli of extruded bovine achilles tendon collagen fibers are reported to be $359.6 \pm 28.4 \mathrm{MPa}[49]$. In [50], the elastic moduli of wet and dry rat tail tendon fibers are reported to be $570 \mathrm{MPa}$ and $2690 \mathrm{MPa}$. The high ratio between the single fiber and ECM stiffness $\left(>10^{4}\right)$ in native biological tissues confirms the 
FE prediction that the pattern strip needs to be much stiffer than the base

560 hydrogel to generate the toe-heel-linear behavior.

In this paper, the base hydrogel is made of $20 \mathrm{kDa}$ PEGDA molecules, and the crosslinks in the pattern stripes are formed between the $3.4 \mathrm{kDa}$ and 20 kDa PEGDA molecules. Although the quantitative results obtained from the numerical simulations only apply to hydrogels fabricated from above PEGDA molecule weights, the numerical strategy presented in this paper can be used to examine the material properties of hydrogels made of other molecule weights.

In the photolithographic patterning process described in [9], the collimated UV light is evenly distributed and the crosslinking density remains constant in the thickness direction of the hydrogel sample. For hydrogels patterned with 2D geometries discussed in this research, the anisotropy caused by material heterogeneousness mainly occurs in the plane formed between the sample length and width directions. Because the material properties do not vary in the thickness direction, 2D formulations should be sufficient to capture the most dominant features of the mechanical behavior of patterned hydrogels under discussion. However, 2D formulations may not be enough to represent a strongly microheterogeneous material. For instance, hydrogels are patterned into a layeredstructure with each layer having a different 2D pattern geometry or properties, see [8]. In this case, the material properties do vary in the thickness direction and a 3D computational framework needs to be used to consider the anisotropy caused by material heterogeneousness in all the three directions.

Though not shown in this paper, the adopted computational strategy is also implemented in 3D. An extra spatial angle is introduced in the random walk algorithm to generate the three-dimensional fiber network structure. A similar projection scheme shown in Fig. $2 \mathrm{~b}$ is used to describe the deformation of a fiber segment by the nodes of a 3D element. The component forms of the element residual and the stiffness matrix contributed by a fiber segment in $3 \mathrm{D}$ take the same forms as shown in Eqs. 8 and 9.

PEGDA hydrogels are designed as scaffolds to encapsulate cells for tissue growth and remodeling. In literature, there are many experimental studies 

theoretical and experimental studies.

\section{Appendix A}

A necessary and sufficient condition for Eq. 6 to be convex is that

$$
\frac{\partial^{2} \Psi_{f}(\lambda)}{\partial \lambda^{2}} \geq 0, \quad \forall \lambda \geq 1
$$

The second derivative of $\Psi_{f}(\lambda)$ can be written as,

$$
\frac{\partial^{2} \Psi_{f}(\lambda)}{\partial \lambda^{2}}=\frac{k_{f} L_{0}^{2}}{2 \lambda^{z_{f}+2}}\left[\left(z_{f}^{2}-3 z_{f}+2\right) \lambda^{2}+\left(2 z_{f}-2 z_{f}^{2}\right) \lambda+\left(z_{f}^{2}+z_{f}\right)\right] .
$$

For the above function to be non-negative, the coefficient of the $\lambda^{2}$ term has to be positive

$$
z_{f}^{2}-3 z_{f}+2>0 \quad \Rightarrow \quad z_{f}>2 \text { or } z_{f}<1
$$

Under the condition shown in Eq. 16, when the discriminant of the quadratic part of Eq. 15 is non-positive, that is,

$$
\left(2 z_{f}-2 z_{f}^{2}\right)^{2}-4\left(z_{f}^{2}-3 z_{f}+2\right)\left(z_{f}^{2}+z_{f}\right)=8 z_{f}^{2}-8 z_{f} \leq 0 \quad \Rightarrow \quad 0 \leq z_{f} \leq 1,
$$

the condition shown in Eq. 14 always holds. Therefore, when $0 \leq z_{f}<1$, the

fiber strain energy function shown in Eq. 6 is always convex.

Recall that fibers cannot resist compression, thus, Eq. 14 only needs to hold when $\lambda \geq 1$.0. The constraint on $z_{f}$ can be relaxed. The two roots of Eq. 15 are

$$
\lambda_{1,2}=\frac{-\left(2 z_{f}-2 z_{f}^{2}\right) \pm \sqrt{8 z_{f}^{2}-8 z_{f}}}{2\left(z_{f}^{2}-3 z_{f}+2\right)}, \quad 8 z_{f}^{2}-8 z_{f}>0
$$


Under the condition shown in Eq. 16, when $\lambda \geq \lambda_{1}$ or $\lambda \leq \lambda_{2}$, the condition shown in Eq. 14 holds. On the other hand, since $\lambda \geq 1$.0, as long as $\lambda_{1} \leq 1.0$, the condition shown in Eq. 14 will hold for $\forall \lambda \in[1.0,+\infty)$. That is,

$$
\lambda_{1}=\frac{-\left(2 z_{f}-2 z_{f}^{2}\right)+\sqrt{8 z_{f}^{2}-8 z_{f}}}{2\left(z_{f}^{2}-3 z_{f}+2\right)} \leq 1.0 \quad \Rightarrow \quad z_{f}<0 .
$$

Finally, when $z_{f}=1$,

$$
\frac{\partial^{2} \Psi_{f}(\lambda)}{\partial \lambda^{2}}=\frac{k_{f} L_{0}^{2}}{\lambda^{3}}>0 .
$$

In summary, in order to guarantee the convexity of the fiber strain energy function shown in Eq. 6, the material parameter $z_{f}$ has to satisfy the constraint $z_{f} \leq 1$.

\section{Acknowledgements}

The authors thank Prof. Jane Grande-Allen and Dr. Xing Zhang for providing the experimental data used in this work and for useful discussions and suggestions. This work was partially funded by NSF, Grant No. 1463432.

\section{References}

[1] V. T. Nkomo, J. M. Gardin, T. N. Skelton, J. S. Gottdiener, C. G. Scott, M. Enriquez-Sarano, Burden of valvular heart diseases: a population-based study, Lancet 368 (9540) (2006) 1005-1011.

[2] M. H. Yacoub, J. J. M. Takkenberg, Will heart valve tissue engineering change the world?, Nature Clinical Practice Cardiovascular Medicine 2 (2) (2005) 60-61.

[3] M. K. Sewell-Loftin, Y. W. Chun, A. Khademhosseini, W. D. Merryman, EMT-inducing biomaterials for heart valve engineering: Taking cues from developmental biology, Journal of Cardiovascular Translational Research 4 (5) (2011) 658-671. 
[4] A. Hasan, K. Ragaert, W. Swieszkowski, T. Selimović, A. Paul, G. CamciUnal, M. R. K. Mofrad, A. Khademhosseini, Biomechanical properties of native and tissue engineered heart valve constructs, Journal of biomechanics 47 (9) (2014) 1949-1963.

[5] T. C. Flanagan, A. Pandit, P. Taylor, S. Jockenhövel, Living artificial heart valve alternatives: A review, European Cells and Materials 6 (2003) 28-45.

[6] C. A. Durst, M. P. Cuchiara, E. G. Mansfield, J. L. West, K. J. GrandeAllen, Flexural characterization of cell encapsulated PEGDA hydrogels with applications for tissue engineered heart valves., Acta biomaterialia 7 (6) (2011) 2467-2476.

[7] E. H. Stephens, C. Durst, J. L. West, K. Grande-Allen, Mitral valvular interstitial cell responses to substrate stiffness depend on age and anatomic region, Acta biomaterialia 7 (1) (2011) 75-82.

[8] H. Tseng, M. L. Cuchiara, C. Durst, M. P. Cuchiara, C. J. Lin, J. L. West, K. Grande-Allen, Fabrication and mechanical evaluation of anatomicallyinspired quasilaminate hydrogel structures with layer-specific formulations, Annals of Biomedical Engineering 41 (2) (2013) 398-407.

[9] X. Zhang, B. Xu, D. S. Puperi, A. L. Yonezawa, Y. Wu, H. Tseng, M. L. Cuchiara, J. L. West, K. J. Grande-Allen, Integrating valve-inspired design features into poly(ethylene glycol) hydrogel scaffolds for heart valve tissue engineering, Acta Biomaterialia 14 (2015) $11-21$. doi:10.1016/j. actbio.2014.11.042.

[10] R. Sodian, S. P. Hoerstrup, J. S. Sperling, D. P. Martin, S. Daebritz, J. E. Mayer, Jr, J. P. Vacanti, Evaluation of biodegradable, three-dimensional matrices for tissue engineering of heart valves, ASAIO Journal 46 (1) (2000) $107-110$.

[11] A. Mol, C. V. C. Bouten, F. P. T. Baaijens, G. Zünd, M. I. Turina, S. P. Hoerstrup, Tissue engineering of semilunar heart valves: Current status 
and future developments, Journal of Heart Valve Disease 13 (2) (2004) $272-280$.

[12] G. C. Engelmayr, Jr, E. Rabkin, F. W. H. Sutherland, F. J. Schoen, J. E. Mayer, Jr, M. Sacks, The independent role of cyclic flexure in the early in vitro development of an engineered heart valve tissue, Biomaterials 26 (2) (2005) 175-187.

[13] G. C. Engelmayr, Jr, D. K. Hildebrand, F. W. Sutherland, J. E. Mayer, Jr, M. S. Sacks, A novel bioreactor for the dynamic flexural stimulation of tissue engineered heart valve biomaterials, Biomaterials 24 (14) (2003) 2523 - 2532. doi:10.1016/S0142-9612(03)00051-6.

[14] M. S. Hahn, J. S. Miller, J. L. West, Three-dimensional biochemical and biomechanical patterning of hydrogels for guiding cell behavior, Advanced Materials 18 (20) (2006) 2679-2684.

[15] M. S. Hahn, L. J. Taite, J. J. Moon, M. C. Rowland, K. A. Ruffino, J. L. West, Photolithographic patterning of polyethylene glycol hydrogels, Biomaterials 27 (12) (2006) 2519-2524.

[16] S. Nemir, H. N. Hayenga, J. L. West, PEGDA hydrogels with patterned elasticity: Novel tools for the study of cell response to substrate rigidity., Biotechnology and bioengineering 105 (3) (2010) 636-644.

[17] N. C. Padmavathi, P. R. Chatterji, Structural characteristics and swelling behavior of poly(ethylene glycol) diacrylate hydrogels, Macromolecules 29 (6) (1996) 1976-1979.

[18] J. K. Tessmar, A. M. Göpferich, Customized PEG-derived copolymers for tissue-engineering applications, Macromolecular Bioscience 7 (1) (2007) 2339.

[19] F. M. Veronese, A. Mero, The impact of PEGylation on biological therapies, BioDrugs 22 (5) (2008) 315-329. 
[20] B. A. Roeder, K. Kokini, J. E. Sturgis, J. P. Robinson, S. L. Voytik-Harbin, Tensile mechanical properties of three-dimensional type I collagen extracellular matrices with varied microstructure, Journal of Biomechanical Engineering 124 (2) (2002) 214-222.

[21] I. Stanciulescu, J. Dolbow, S. Zauscher, Computational modeling of surface phenomena in soft-wet materials, International Journal of Solids and Structures 46 (6) (2009) 1334-1344.

[22] J.-Y. Sun, X. Zhao, W. R. K. Illeperuma, O. Chaudhuri, K. H. Oh, D. J. Mooney, J. J. Vlassak, Z. Suo, Highly stretchable and tough hydrogels., Nature 489 (7414) (2012) 133-136.

[23] J. A. Stella, M. Sacks, On the biaxial mechanical properties of the layers of the aortic valve leaflet, Journal of Biomechanical Engineering 129 (5) (2007) 757-766.

[24] J. C. Culver, J. C. Hoffmann, R. A. Poché, J. H. Slater, J. L. West, M. E. Dickinson, Three-dimensional biomimetic patterning in hydrogels to guide cellular organization, Advanced Materials 24 (17) (2012) 2344-2348.

[25] A. D'Amore, J. A. Stella, W. R. Wagner, M. Sacks, Characterization of the complete fiber network topology of planar fibrous tissues and scaffolds, Biomaterials 31 (20) (2010) 5345-5354.

[26] Q. Liu, Z. Lu, Z. Hu, J. Li, Finite element analysis on tensile behaviour of 3D random fibrous materials: Model description and meso-level approach,

[27] A. D'Amore, N. Amoroso, R. Gottardi, C. Hobson, C. Carruthers, S. Watkins, W. R. Wagner, M. Sacks, From single fiber to macro-level mechanics: A structural finite-element model for elastomeric fibrous biomaterials, Journal of the mechanical behavior of biomedical materials 39 (2014) 146-161. 
[28] K. Misof, G. Rapp, P. Fratzl, A new molecular model for collagen elasticity based on synchrotron X-ray scattering evidence, Biophysical Journal 72 (3) (1997) 1376-1381.

[29] L. Zhang, S. P. Lake, V. K. Lai, C. R. Picu, V. H. Barocas, M. S. Shephard, A coupled fiber-matrix model demonstrates highly inhomogeneous microstructural interactions in soft tissues under tensile load., Journal of Biomechanical Engineering 135 (1) (2013) 011008-011008.

[30] T. Jin, I. Stanciulescu, Numerical simulation of fibrous biomaterials with randomly distributed fiber network structure, Biomechanics and Modeling in Mechanobiology (2015) 1-14doi:10.1007/s10237-015-0725-6.

[31] G. A. Holzapfel, T. C. Gasser, R. W. Ogden, A new constitutive framework for arterial wall mechanics and a comparative study of material models, Journal of Elasticity 61 (1-3) (2000) 1-48.

[32] D. Balzani, P. Neff, J. Schröder, G. A. Holzapfel, A polyconvex framework for soft biological tissues. Adjustment to experimental data, International Journal of Solids and Structures 43 (20) (2006) 6052-6070.

[33] A. Pandolfi, M. Vasta, Fiber distributed hyperelastic modeling of biological tissues, Mechanics of Materials 44 (2012) 151-162.

[34] R. Taylor, FEAP, a Finite Element Analysis Program: Version 8.3 User Manual, University of California, Berkeley. Dept. of Civil and Environmental Engineering (2011).

[35] Y. Lanir, Constitutive equations for fibrous connective tissues, Journal of biomechanics 16 (1) (1983) 1-12.

[36] F. C. MacKintosh, J. Käs, P. A. Janmey, Elasticity of semiflexible biopolymer networks, Physical Review Letters 75 (24) (1995) 4425-4428.

[37] B. Agoram, V. H. Barocas, Coupled macroscopic and microscopic scale modeling of fibrillar tissues and tissue equivalents, Journal of Biomechanical Engineering 123 (4) (2001) 362-369. 
[38] C. Storm, J. J. Pastore, F. C. MacKintosh, T. C. Lubensky, P. A. Janmey,

[39] D. S. Puperi, A. Kishan, Z. E. Punske, Y. Wu, E. Cosgriff-Hernandez, J. L. West, K. J. Grande-Allen, Electrospun polyurethane and hydrogel composite scaffolds as biomechanical mimics for aortic valve tissue engineering, ACS Biomaterials Science \& Engineering 2 (9) (2016) 1546-1558.

[40] L. Annovazzi, F. Genna, An engineering, multiscale constitutive model for fiber-forming collagen in tension, Journal of Biomedical Materials Research - Part A 92 (1) (2010) 254-266.

[41] G. A. Holzapfel, R. W. Ogden, On the bending and stretching elasticity of

[42] G. A. Holzapfel, R. W. Ogden, Elasticity of biopolymer filaments, Acta biomaterialia 9 (7) (2013) 7320-7325.

[43] I. Arganda-Carreras, R. Fernández-González, A. Muñoz-Barrutia, C. OrtizDe-Solorzano, 3D reconstruction of histological sections: Application to mammary gland tissue., Microscopy Research and Technique 73 (11) (2010) 1019-1029.

[44] G. C. Reilly, A. J. Engler, Intrinsic extracellular matrix properties regulate stem cell differentiation, Journal of Biomechanics 43 (1) (2010) 55 - 62, special Issue on Cell Mechanobiology.

[45] A. J. Engler, L. Richert, J. Y. Wong, C. Picart, D. E. Discher, Surface probe measurements of the elasticity of sectioned tissue, thin gels and polyelectrolyte multilayer films: Correlations between substrate stiffness and cell adhesion, Surface Science 570 (2004) 142-154. doi:10.1016/j. susc.2004.06.179.

750

[46] A. M. Collinsworth, S. Zhang, W. E. Kraus, G. A. Truskey, Apparent elastic modulus and hysteresis of skeletal muscle cells throughout differentiation, 
American Journal of Physiology - Cell Physiology 283 (4) (2002) C1219C1227. doi:10.1152/ajpcell.00502.2001.

[47] A. J. Engler, S. Sen, H. L. Sweeney, D. E. Discher, Matrix elasticity directs stem cell lineage specification, Cell 126 (4) (2006) 677 - 689. doi:10.1016/ j.cell.2006.06.044.

[48] P. Dutov, O. Antipova, S. Varma, J. P. R. O. Orgel, J. D. Schieber, Measurement of elastic modulus of collagen type I single fiber, PLoS ONE 11 (1). doi:10.1371/journal.pone.0145711.

[49] E. Gentleman, A. N. Lay, D. A. Dickerson, E. A. Nauman, G. A. Livesay, K. C. Dee, Mechanical characterization of collagen fibers and scaffolds for tissue engineering, Biomaterials 24 (21) (2003) 3805-3813. doi:10.1016/ S0142-9612(03) 00206-0.

[50] Y. Kato, D. L. Christiansen, R. A. Hahn, S.-J. Shieh, J. D. Goldstein, F. H. Silver, Mechanical properties of collagen fibres: a comparison of reconstituted and rat tail tendon fibres, Biomaterials 10 (1) (1989) $38-42$. doi:10.1016/0142-9612(89)90007-0.

[51] H. Tseng, D. S. Puperi, E. J. Kim, S. Ayoub, J. V. Shah, M. L. Cuchiara, J. L. West, K. J. Grande-Allen, Anisotropic poly(ethylene glycol)/polycaprolactone hydrogelfiber composites for heart valve tissue engineering, Tissue Engineering. Part A 20 (19-20) (2014) 2634-2645.

[52] D. S. Puperi, L. R. Balaoing, R. W. O'Connell, J. L. West, K. J. GrandeAllen, 3-dimensional spatially organized peg-based hydrogels for an aortic valve co-culture model, Biomaterials 67 (2015) 354 - 364. doi:10.1016/ j.biomaterials.2015.07.039. 


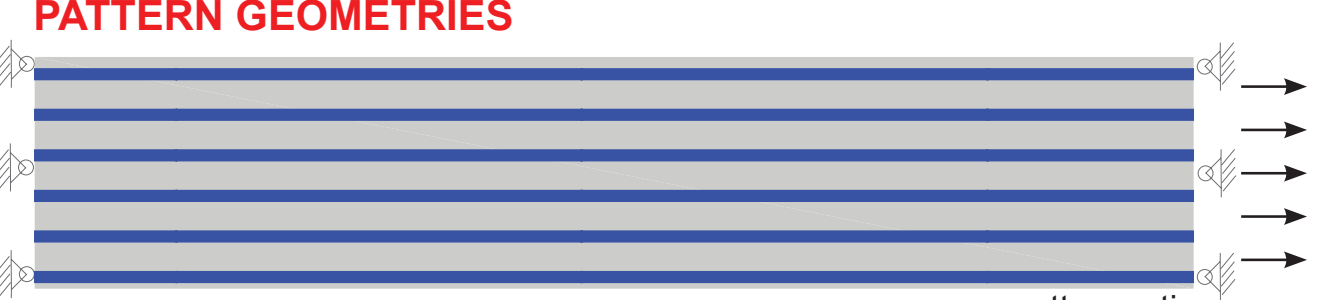

pattern ratio

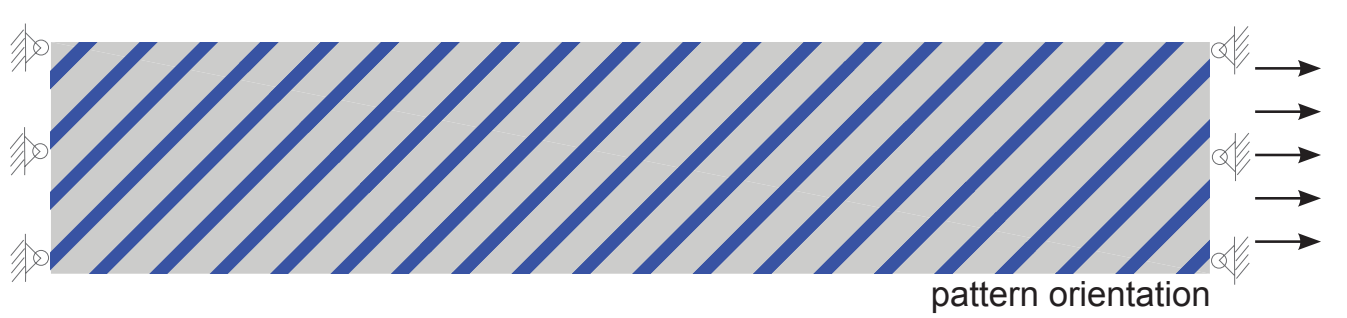

$\stackrel{2}{\stackrel{4}{\longrightarrow} \text { 和 }}$

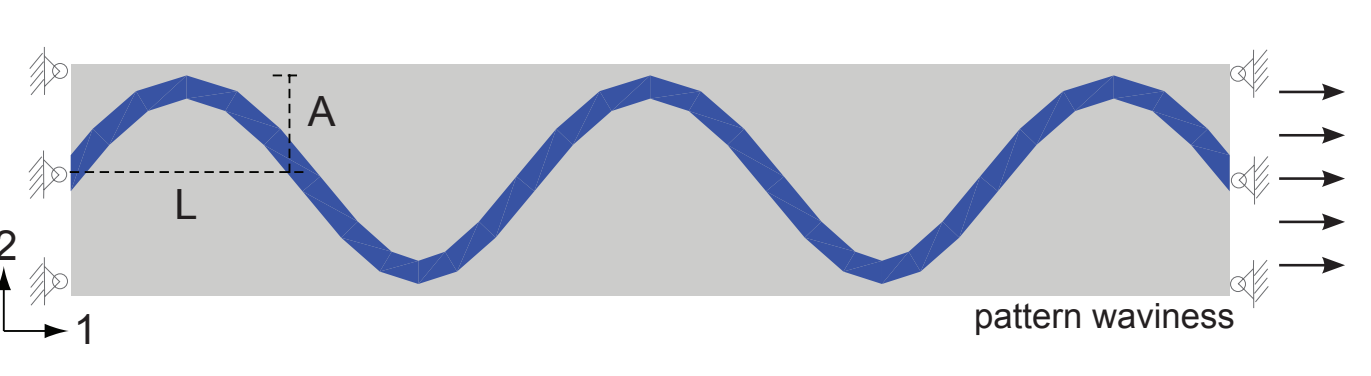

EXPERIMENTS

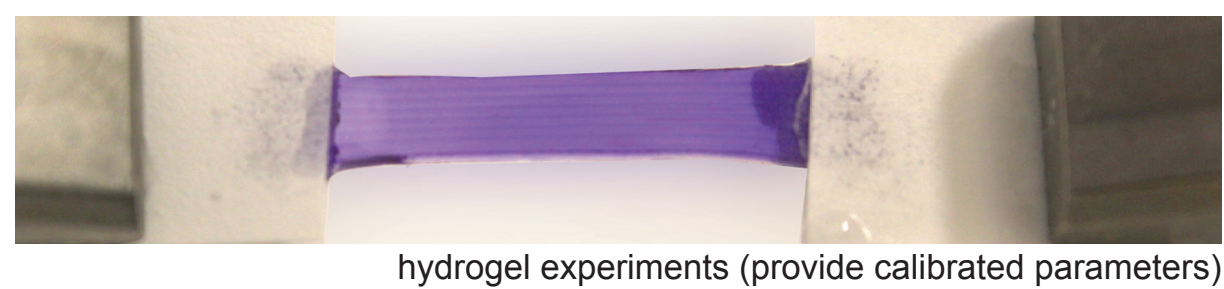

hydrogel experiments (provide calibrated parameters) $\downarrow$

\section{PATTERN STRIPE} BASE HYDROGEL

NUMERICAL RESULTS AND PREDICTIONS

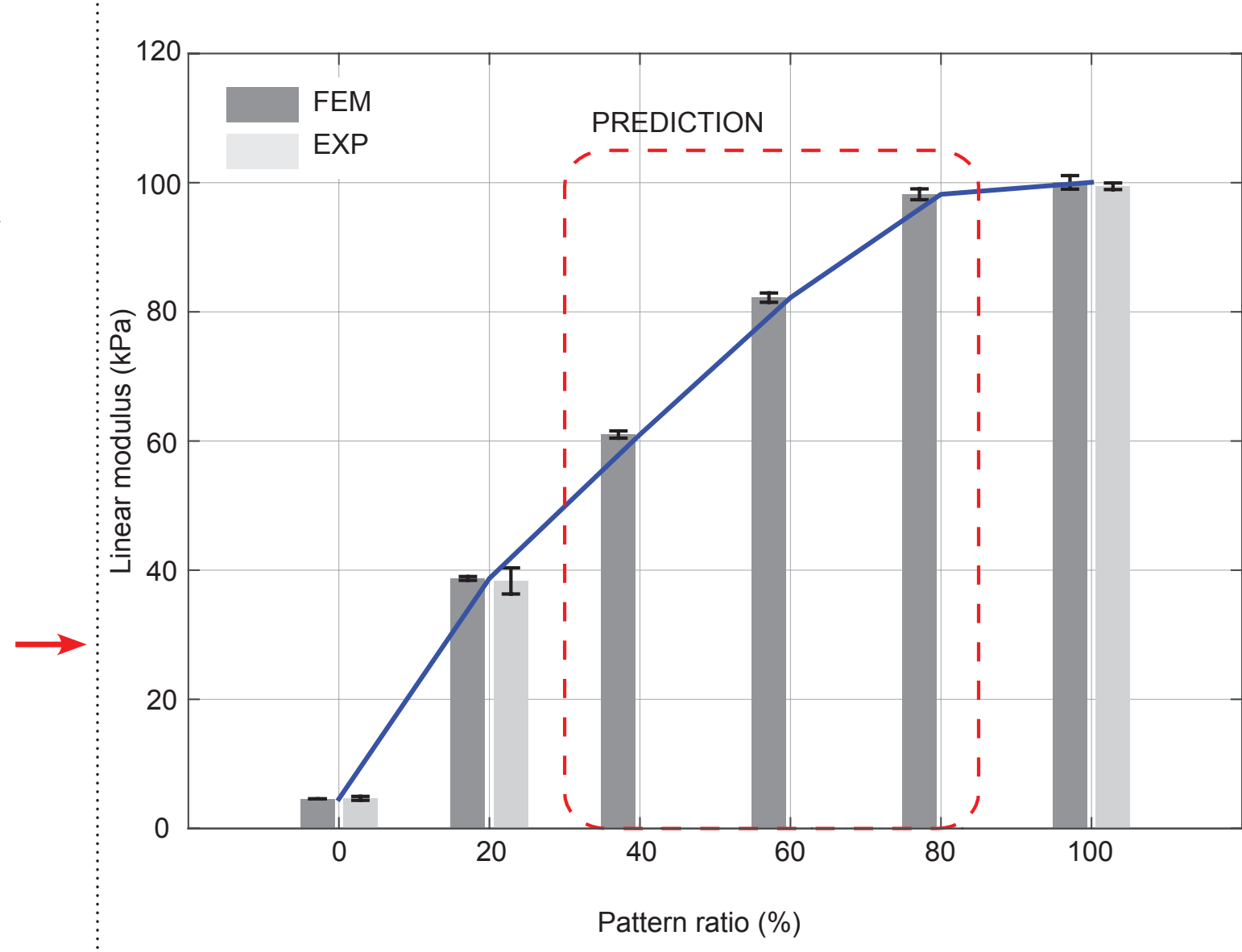

